\title{
Excitonic Crystal, Nanotechnology and New Prospect for Optoelectronics
}

\author{
Sergei L. Pyshkin*
}

Institute of Applied Physics, Academy of Sciences, Kishinev, Moldova

\begin{abstract}
The review demonstrates the results of development of growth technology for perfect and free of contamination gallium phosphide $(\mathrm{GaP})$ crystals and investigation of influence of crystallization conditions on quality and properties of the crystals. The long-term ordered and therefore close to ideal crystals repeat behavior of the best nanoparticles with pronounced quantum confinement effect. These perfect crystals are useful for application in top-quality optoelectronic devices as well as they are a new object for development of fundamentals of solid state physics.

Since the time of original preparation by the author in the 1960s of gallium phosphide crystals doped by nitrogen $(\mathrm{GaP}: \mathrm{N})$, followed by the introduction of the excitonic crystal concept in the 1970s, the best methods of bulk, film and nanoparticle crystal growth were elaborated. The results of semi centennial evolution of GaP:N properties are compiled in the paper. Novel and useful properties of perfect $\mathrm{GaP}$ including its stimulated emission, very bright and broadband luminescence at room temperature were observed. These results provide a new approach to selection and preparation of perfect materials for optoelectronics and a unique opportunity to realize a new form of solid-state host - the excitonic crystal as high intensity light source with expected low threshold for generation of non-linear optical effects.

Using the example of $\mathrm{GaP}$, here is proposed the cheap, resource-saving and impactful way for development of optoelectronics with the help of a special transformation of an ordinary semiconductor into the base material for various device structures.
\end{abstract}

Keywords: GaP, long-term ordering, excitonic crystal, perfect semiconductors for optoelectronics.

\section{INTRODUCTION}

Single crystals of semiconductors grown under laboratory conditions naturally contain a varied assortment of defects such as displaced host and impurity atoms, vacancies, dislocations, and impurity clusters. These defects result from the relatively rapid growth conditions and inevitably lead to the deterioration of mechanical, electric, and optical properties of the material, and therefore to degradation in performance of the associated devices. Note, the deterioration of optical properties of an luminescent material for application in optoelectronics may appear in the complete absence of light emission in the spectral region, where the perfect material gives an excellent luminescence, in very narrow emissive band, in a very weak light emission, in impossibility to control its shape and brightness, as well as in fast degradation of a device prepared on the base of this material. In order to partly overcome the noted above difficulties industry uses expensive, but only palliative decisions, such as limitless extension of the list of materials for the device making or small improvement of technologies for growth and preparation of electronic materials. Huge material, time and mental resources already have spent and will be spent further in our efforts to support or improve achieved parameters and reliability of electronic devices. Therefore finding alternative

*Address correspondence to this author at the Institute of Applied Physics, Academy of Sciences, Kishinev, Moldova; Fax: (37322) 738149;

Tel: (373) 079607919; E-mail: spyshkin@yahoo.com drastic methods for device making is one of the main priorities of electronic industry development. This paper describes the experience of the author in this field.

The pure and doped GaP crystals discussed herein were prepared about 50 years ago [1]. Throughout the intervening decades they have been periodically re-evaluated in order to investigate changes over time in their electro- and photoluminescence, photoconductivity, as well as changes in bound excitons characteristic for doped $\mathrm{GaP}$ and other phenomena. Accordingly, it was of interest also to monitor the change in crystal quality over the course of several decades while the investigated crystals are held under ambient conditions.

Over time, as it is confirmed by 50 years of the relevant experiments, driving forces such as diffusion along concentration gradients, strain relaxation associated with clustering, and minimization of the free energy associated with properly directed chemical bonds between host atoms result in an ordered redistribution of impurities and host atoms in a crystal. In the particular case of $\mathrm{GaP}$ and some other chemical compounds, having in their compositions highly volatile components, any attempt to accelerate these processes through annealing at increased temperatures cannot be successful. That is because high-temperature processing results in thermal decomposition (in GaP - due to P desorption) instead of improved crystal quality. Therefore successful thermal processing of these compounds can only take place at temperatures below the sublimation temperatures of their 
volatile constituents, requiring a longer annealing time. For instance, as evaluated in the framework of the Ising model, the characteristic time of the substitution reaction during $\mathrm{N}$ diffusion along $\mathrm{P}$ sites in $\mathrm{GaP}: \mathrm{N}$ crystals at room temperature constitutes 15-20 years [2]. Hence, the observations of highly excited luminescence and some other phenomena in the crystals made in the 1960s-1970s and in the 1980s-1990s were then compared with the results obtained in 2005-2014 under similar experimental conditions.

The long-term ordering of doped $\mathrm{GaP}$ and other semiconductors has been observed as an important accompanying process, which only can be studied using the same unique set of samples and the interest to observe them over decade time scales. More specifically, the optical and mechanical properties of single crystalline GaP and some other semiconductors, also grown in the 1960s, have been analyzed. Comparison of the properties of the same crystals was performed in the 1960s, 1970s, 1980s, 1990s [1, 3-17] along with those of newly made GaP nanocrystals [18-20] and freshly prepared bulk single crystals [21-24]. Jointly with the references [25, 26] this review provides generalization of the results on long-term observation of luminescence, absorption, Raman light scattering, and microhardness of the bulk single crystals in comparison with the same properties of the top quality $\mathrm{GaP}$ nanocrystals. It is shown that the combination of these characterization techniques elucidates the evolution of these crystals over the course of many years. It clarifies the ordered state brought about by prolonged room-temperature thermal annealing, and the interesting optical properties that accompany such ordering. Demonstrated is that long-term natural stimuli that improve the perfection of crystals prevail over other processes and can lead to novel heterogeneous device systems and new semiconductor devices with high temporal stability.

Additionally, it is worth noting, that semiconductor nanoparticles for optoelectronic applications also were synthesized mainly to avoid limitations inherent to freshly grown bulk semiconductors with a wide range of different defects. For instance, different defects of high concentration in freshly prepared $\mathrm{GaP}$ single crystals completely suppress any luminescence at room temperature due to the negligible free path for non-equilibrium electron-hole pairs between the defects and their non-radiative recombination, while the quantum theory predicts their free movement in the field of an ideal crystal lattice. The long-term ordered and therefore close to ideal crystals demonstrate equivalent to the best nanoparticles bright luminescence and stimulated emission. These perfect crystals, due to their unique mechanical and optical properties, are useful for application in high-quality optoelectronic devices as well as they are a new object for development of fundamentals of solid state physics, nanotechnology and crystal growth.

Also noted is the application of $\mathrm{GaP} /$ polymers nanocomposites in device structures for accumulation, conversion and transport of light energy has only recently received attention while bulk and thin GaP films have been successfully commercialized for many years. Therefore, for completeness, during the recent years, since 2005, the author and his colleagues have continued their efforts on the preparation of $\mathrm{GaP}$ nanoparticles in order to improve their quality and to apply their composites with appropriate polymers for advanced light emissive structures [18-20, 27-31].

In preparing this review, the author did not intent to evaluate the works on $\mathrm{GaP}$ of the other authors, but their works are cited and used here when it is necessary for explanation and interpretation of new phenomena observed during longterm ordering of impurities and host atoms in the crystal lattice. Elaborating optimal methods of preparation of GaP bulk crystals, nanoparticles and their light emissive composites with compatible polymers we use our own experience and literature data [32-34].

The main goals of these 50 years efforts, and of this review, are, firstly, observation and description of very interesting results of the long-term evolution of GaP properties. Secondly, resulting from this experience, a proposal is made of alternative and resource-saving way for development of electronics for many years ahead. The excitonic crystal [26] is proposed and justified as new optical media for the future optoelectronic devices used in optical data processing, storage and transmission as well as for generation of non-linear optical effects at rather modest thresholds for nonlinearities. Interesting and very useful for application results of longterm evolution of $\mathrm{GaP}$ properties as well as the unique collection of tested and stored for years pure and doped perfect $\mathrm{GaP}$ crystals are demonstrated and proposed to academic researchers, engineers and managers of electronic industry for intensification of collaboration in patent activity, reorganization of the material and device making processes, reduction in price, improvement of parameters and reliability of devices.

The first wide presentation of the works on growths of perfect GaP crystals (1963) started in A.F. Joffe PhysicoTechnical Institute and State Polytechnical University, St. Petersburg, Russia, took place at the 7th International Crystallography Congress (Moscow, July 1966) [1]. Investigations of properties and evolution of these crystals since the moment of their preparation and until present time were conducted mainly in research and industrial centers of Moldova (Institute of Applied Physics, Academy of Sciences), Russia (Ioffe Institute, Lomonosov Moscow State University and Ac. Scie. Institute of General Physics, Moscow), USA (Clemson University, SC, and University of Central Florida, Orlando, FL) and Italy (Universita di Roma "Sapienza", Istituto di elettronica dello stato solido, CNR, Rome, Universita degli studi di Cagliari, Università degli studi di Milano). The results on growth of the crystals and investigation of their properties have been discussed during the entire period of 1966-2014 at many international conferences, most frequently, at the conferences in framework of the US MRS and TMS Societies. They have also been regularly published in relevant scientific literature, proved by the Author's $\mathrm{PhD}$ [3] and D.Sc. [7] dissertations and used in joint projects with the former USSR academic institutions, defence industry and electronic ministries. Later results were also used in US Air Force and Navy Research Offices, within scientific grants of the US Department of States, Civil Research Development, Soros and Fulbright Foundations, Italian Consiglio 


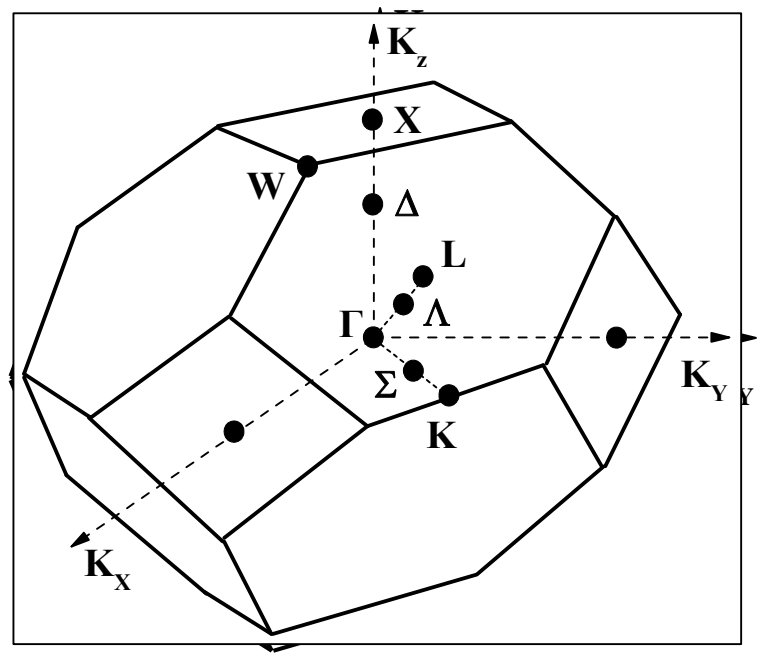

Fig. (1). Brillouin zone of Gallium Phosphide.

Nazionale delle Ricerche. Notably, all suggestions on preparation and properties of materials, proposed by the Author for electronic industry, have always been accepted with big interest of audience, while constructive criticism contained a lot of interesting and useful refinements and proposals. The work on improvements and a lot of reviewers remarks lasted for years since the first public discussions in 1963. Such long-term and fruitful semi-centennial discussions and investigations resulted into proven statements and results carefully elaborated and tested during the years.

This activity was stimulated and followed by the new and extremely interesting natural processes and phenomena elapsed with time in the crystals.

This review is divided into six parts, respectively structured around the following items:

1) Properties and characteristics of GaP;

2) Growth technology for perfect and free of contamination $\mathrm{GaP}$ crystals;

3) Optical properties of perfect, long-term ordered GaP:N in comparison with freshly prepared crystals;

4) Long-Term Convergence of Bulk- and Nano-Crystal Properties of $\mathrm{GaP}$; ics;

5) Excitonic crystal and its importance for optoelectron-

6) Conclusions.

\section{PROPERTIES OF GAP}

$\mathrm{GaP}$ crystallizes in zinc blende structure, where $\mathrm{Ga}$ and $\mathrm{P}$ atoms create two interpenetrating face-centered lattices spaced $1 / 4$ of the (111) cube diagonal apart.

Brillouin zone of GaP and the other III-V compounds represents the truncated octahedron (Fig. 1) having the following high symmetry points:
$\Gamma-\bar{K}=(000)-$ center of Brillouin zone,

$\Delta$ - along the (100) axises inside the zone,

$\mathrm{X}$ - (100) Brillouin zone edge,

$\sum$-along the (110) axises inside the zone,

$\mathrm{K}$ - (110) Brillouin zone edge,

$\Lambda$ - along the (111) axises inside the zone,

$\mathrm{L}$ - (111) Brillouin zone edge.

A concrete band structure for each III-V representative in the limits of common for them Brillouin zone depends on the type of symmetry of the wave functions of valence electrons of the atoms, creating the compound. The most reliable data on $\mathrm{GaP}$ band structure were obtained from the experiments on light absorption and reflection as well as using the spectral distribution of photoconductivity in the region of intrinsic absorption.

According to W. Paul empirical rule [35] the energy gaps equally depend on the pressure for the relevant electron states. Using this rule and experiments on dependence of electron transitions on pressure, the authors of [36] have proposed the band structure of $\mathrm{GaP}$ presented in Fig. (2).

Experimental data confirm the details of the GaP band structure. So, the absolute minimum of the conductance band (the X-point) presented in Fig. (1) lays at the edge of Brillouin zone in the (100) direction, while the valence band maximum (the $\Gamma$-point) position is the center of the zone. The absolute minimum value of the forbidden gap (Fig. 1) corresponds to the indirect optical transition $\Gamma_{15}{ }^{\mathrm{v}} \rightarrow \mathrm{X}_{1}^{\mathrm{c}}$; this value depends on the temperature changing between 2,354 $\mathrm{eV}$ at $4,2^{\circ} \mathrm{K}$ (liquid helium) and 2,328 at $77^{\circ} \mathrm{K}$ (liquid nitrogen) until $2,248 \mathrm{eV}$ at $300^{\circ} \mathrm{K}$ (room temperature) [36]. Minimum gap for direct optical transition $\Gamma_{15}{ }^{\mathrm{v}} \rightarrow \Gamma_{1}^{\mathrm{c}}$ at $300^{\circ} \mathrm{K}$ is equal to $2,78 \mathrm{eV}$ [36]. The valence band, taking into account its spin-orbit splitting, consists of two confluent bands and another one shifted downward. The other details of $\mathrm{GaP}$ band structure are shown in Fig. (2). 


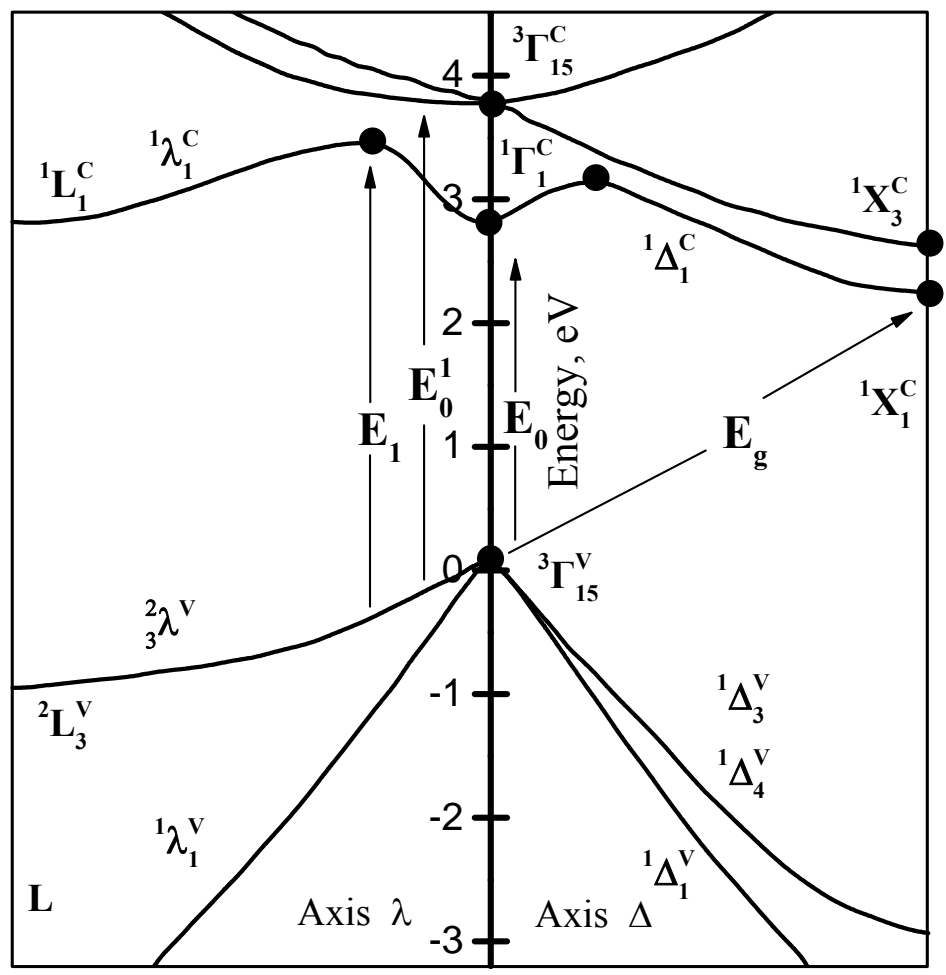

Fig. (2). Band structure of Gallium Phosphide.

According to the quantum selection rules for optical transitions, the lattice phonons do not participate in the direct transitions, while in the indirect transition participate lattice phonons The type and energy of these phonons are determined in [37] together with the low- and high-frequency dielectric constants, 10,182 and 8,457 respectively. The data on $\mathrm{GaP}$ phonon spectrum are widely used to interpret its light emissive and absorption spectra. Transversal and longitudinal acoustic and optic phonons with the energies 12,8 (TA), 31,3 (LA), 46,5 (LO), 50,0 meV (TO) participate in indirect optical transitions [37-39]. Note, at low temperatures, when the thermal energy $\mathrm{kT}$ is less than the respective energies of the free and bound exciton creation (10 and 21 meV respectively for free and $\mathrm{N}$ bound excitons $[4,7])$, the indirect optical transitions occur mainly through the excitonic states.

\section{GROWTH TECHNOLOGY FOR PERFECT GAP CRYSTALS}

Single crystals of gallium phosphide, in principle, can be obtained in several ways $[1,3,7]$. Method for obtaining gallium phosphide from solution-melt, chosen by us, has several significant advantages:

1) Significant temperature reduction of the process and the presence of large amounts of solvent dramatically reduce crystal pollution by material of the container;

2) The light sources created on their basis have high efficiency;
3) Due to specifics of the method, gallium phosphide at the appropriate level of the experiment can be obtained in the form of the relatively large lamellar crystals of a definite crystallographic orientation. Note, lamellar crystals are the most convenient and economically-sound material in manufacturing of many semiconductor devices.

Consider peculiarities of growth of lamellar crystals of gallium phosphide with the set-up properties, formed under uniform cooling of the $\mathrm{P}$ solution in Ga. The influence of the following factors has been investigated: 1) quantity and chemical nature of impurities; 2) geometric shape of the container and conditions of heat rejection; 3 ) accuracy of the temperature control; 4) cooling rate and the law of temperature change in time.

The aim of this work was to elucidate the mechanism of crystallization of lamellar GaP crystals and to conform through experiments stated earlier assumptions about the nature of this process.

I. The solution-melt method of GaP growth is described in detail in $[1,3,7]$. So we accentuate here only the features of crystal growth specific for this study associated.

Synthesis, alloying and crystallization were combined in a single cycle. The process was conducted in a sealed and drained container from optical quartz that was flushed with spectral pure argon before being pumped. Experiments on the establishment of the influence of impurities on growth of $\mathrm{GaP}$ crystals was performed using $\mathrm{Ga} 000$ and phosphorus B5, industrially prepared in former USSR, the highest purity components produced at that time (the $1960^{\text {th }}$ ). The impact of 


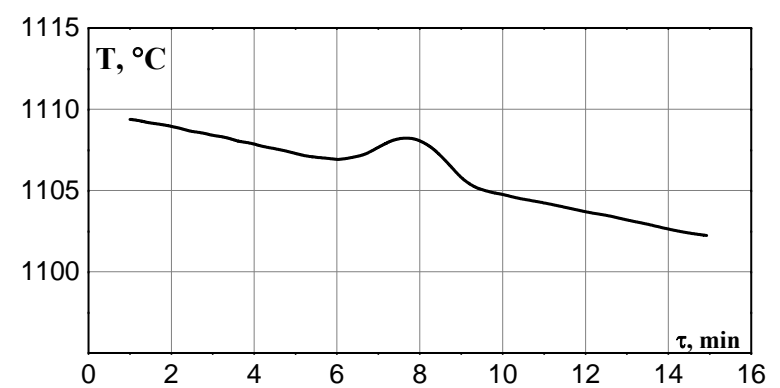

Fig. (3). Change of temperature in the container when cooling with $30 \mathrm{deg} /$ hour the 5 at.\% solution of $\mathrm{P}$ in $\mathrm{Ga}$.

the temperature control accuracy was investigated at the facility, which allows to reproduce with controlled accuracy in the growth container the necessary law of the temperature change $[1,3]$. Permissible fluctuation of temperature can be set within $\pm 0,5 \pm 10^{\circ} \mathrm{C}$ interval. In order to study the form and quality of growing crystals, the crystallization process could be interrupted at any temperature below the point of liquidus. This interruption was achieved with the help of a special designed growth container and its programmable heating furnace, which allow to stop the change of temperature of the solution-melt, to identify and examine grown by this time the crystals (see details in $[1,3]$ ).

II. With the introduction into the $\mathrm{Ga}$ solvent of various impurities, the choice of which was determined by important for application properties of the obtained crystals, the effects of contaminants on the nature of solidification have been investigated at the same time. The following impurities were introduced one by one in the solvent: $\mathrm{Cu}, \mathrm{Zn}, \mathrm{Cd}, \mathrm{In}, \mathrm{Si}, \mathrm{Ge}$, $\mathrm{Sn}, \mathrm{S}, \mathrm{Se}, \mathrm{Te}, \mathrm{Ni}, \mathrm{Cr}, \mathrm{Fe}, \mathrm{Co}, \mathrm{Sm}, \mathrm{La}, \mathrm{Gd}$ and $\mathrm{Sm}_{2} \mathrm{O}_{3}$.

Typical features of the doped GaP crystals (size, color, polarity, lamellar structure, twinning, the dislocation structure and others) were compared with similarly grown, but undoped, crystals.

It was found that the properties of the crystals depend on the chemical nature as well as on the quantity of the selected impurities. Their small admixtures did not significantly change the form of the crystals, while significant change of the crystallization environment composition created changes in the forms of growth. For instance, introduction into the solution of large quantities of $\mathrm{Sn}$, the element of another valence compared with $\mathrm{Ga}$, led to the emergence of the crystals having octahedral isometric forms. Isometric forms with simultaneous reduction of the sizes of crystals occurred with the introduction of solvent refractory impurities - samarium oxide $\left(\mathrm{Sm}_{2} \mathrm{O}_{3}\right)$, solid units of which, apparently, were the centers of crystallization. Introduction into the solution of significant quantities of In, the chemical analogue of $\mathrm{Ga}$, only slightly influenced on the morphology of the crystals, changing the lattice parameter, indicating the formation of 0.01 at. \% In-GaP solid solution.

It was established that an increase in concentration of $\mathrm{Zn}$ and Gd in the Ga-P solution leads to the increase in the concentration of carriers (holes), reducing the size of crystals and their chemical resistance with a simultaneous increase in microhardness on the plane (111). At introduction of S (1 at.
$\%$ and more) the forms of the crystals varied from lamellar to volumetrical. By increasing the concentration of tellurium in solution from 0.007 to 0.02 at. $\%$ the lattice constant varied from 5.4511 to $5.4524 \AA$.

At small quantities of impurities $\mathrm{Zn}$ and $\mathrm{Te}$ in $\mathrm{GaP}$, until about $10^{19} \mathrm{~cm}^{-3}$, a direct proportional relationship is observed between the amount of impurities added to the solution and the concentration of charge carriers in the crystals.

Doping of the Ga-P solution by small quantities of rare earths and elements of the Fe group did not impact significantly the forms of crystals. It, however, led to a significant change in the electrical and luminescent properties of $\mathrm{GaP}$ crystals.

It is interesting to note that the density of etching pits on the plane (111) of doped crystals sharply increased in comparison with the undoped ones.

III. The following factors influenced the morphology of $\mathrm{GaP}$ crystals grown from the Ga-P solution of a certain concentration in conditions of the mass crystallization: the degree of supersaturation, which depends on the temperature of the solution and its first time derivative; the conditions of the heat crystallization removal and the accuracy of the temperature control in the solution.

Changing conditions of crystallization, mainly the rate of cooling of the melt, significantly affects the morphology of crystals. So, the increase of the cooling rate reduced the size of the crystals, but the dislocation density had not changed and was primarily determined by concentration and chemical nature of impurities. The number of crystals having isometric forms increased with decreasing of cooling rate at simultaneous improvement of quality of the crystal surface. Uneven removal of heat from walls of the container led to the formation of up to $30 \mathrm{~mm}$ crystal needles, elongated in the direction of the heat removal.

More accurate temperature control provides significant impact on the quality of the crystal. The crystals obtained with the accuracy of temperature control $\pm 0.5^{\circ} \mathrm{C}$ had a perfect planes (111), low dislocation density and dimensions in 2-3 larger than the crystals obtained at the same conditions, but with the accuracy $\pm 5^{\circ} \mathrm{C}$ or according to the regimes described in the literature. Crystals reached $25 \mathrm{~mm}$ in length and had the dislocation density $\sim 10^{3} \mathrm{~cm}^{-2}$, which is significantly less than that of the crystals obtained with low accuracy of the temperature control.

IV. In order to clarify the mechanism of crystallization of lamellar GaP crystals, differential record of temperature change between the standard of pure $\mathrm{Ga}$ and 5 at.\% Ga-P solution near the point of liquidus as well as interruption of the crystallization process at different temperatures below the point of liquidus were conducted in special containers described in [1]. Temperature was recorded in the locations of the Ga standard and the Ga-P solution. Interruption of the process was carried out by turning the container with quartz net. The pattern of temperature change in the solution-melt is presented in Fig. (3).

The following describes an overview of the growth of lamellar GaP crystals on the basis of the experiments conducted. Total quantity of the solution-melt in the experi- 
ments was $10-12 \mathrm{~cm}^{3}, 1 \mathrm{~cm}^{3}$ of this solution ( 5 at. $\%$ of $\mathrm{P}$ ) at a temperature above the line of the liquidus contained $3 \times 10^{21}$ and $6 \times 10^{22}$ of $\mathrm{P}$ and $\mathrm{Ga}$ atoms respectively.

The first crystals, having the form of dendritic needles with the length of the order of $5 \mathrm{~mm}$ and thickness of 0,15 $\mathrm{mm}$, were registered at the temperature $1107^{\circ} \mathrm{C}$ at approximately $16^{\circ} \mathrm{C}$ supercooling. The crystals, found on the net inside the growth container [1] at lower temperatures of the process interrupting, represent thin plates in the form of rhombs, triangles or hexagons. Twinning on the transverse cross-sections was observed upon the examination of microsections and cleaved facets. It turned out that the lamellar $\mathrm{GaP}$ crystals contain the twinning plane, parallel to the planes (111).

Growth process of lamellar crystals can be formally divided into two components: the tangential growth in the plane (111) and layer-by-layer growth in the direction normal to the plane (111). Because the ratio of $\mathrm{Ga}$ and $\mathrm{P}$ atoms differed significantly from the stoichiometric, it is natural to assume that diffusion determines the rate of growth. According to our estimates, the coefficient of diffusion of phosphorus atoms in $\mathrm{Ga}$ at $\mathrm{T} \sim 1400 \mathrm{~K}$ is equal $\mathrm{D}_{\mathrm{p}} \sim 3 \times 10^{-3} \mathrm{~cm}^{2} \mathrm{sec}^{-1}$.

Upon cooling system to $1107^{\circ} \mathrm{C}$ the supercooling of the solution was approximately $1.6 \times 10^{20} \mathrm{~cm}^{-3}$. Accepting the growth of needle-shaped crystals ends during recalescence time about 2 min (Fig. 3), we get the diffusion length $\mathrm{L}=$ $\left(D_{\mathrm{p}} \tau\right)^{1 / 2}=0.6 \mathrm{~cm}$. The dimensions of the crystal in diffusion approximation become: needle length $6 \mathrm{~mm}$ and thickness $0.56 \mathrm{~mm}$; this value is in agreement with the experimental data. With further lowering of the temperature, the crystals acquire lamellar form with developed planes (111) having the greatest reticular density and composed of the same atoms. Note that the removal of heat of crystallization is facilitated by lamellar form of the crystals.

Thus, accepting the noted above two-stage model of lamellar crystal growth, let us consider the ratio of the contributions of the tangential growth in the plane (111) and layerby-layer growth in the direction normal to the plane (111). The growth in the tangential direction is limited by diffusion and by the size of the previous layer, i.e., is determined by the initial conditions and the shape and size of the plates, formed during the cooling of the solution-melt 10-20 degrees below the point of liquidus. Since the formation of the initial crystals occurs in a very short time, the growth in the tangential direction is difficult to control, as it is in the case of the dendritic crystal growth. The growth in the direction normal to the plane (111) is sensitive to the fluctuations of the degree of supersaturation, resulting from the thermal motion of atoms and temperature fluctuations. In general, the better uniformity and perfection of the surface of lamellar GaP crystals, the less the time ratio needed for flat nucleation center formed a new layer to the time for which will be created the next nucleation center. The latter, obviously, in an extreme extent depends on fluctuations of the degree of supersaturation in different points of the growing plane and at different points in time. With the deterioration of precision temperature control the number of fluctuations increases and bad crystals grow in the conditions of "entanglement" of the above noted characteristic times. Small fluctuations of supersaturation also are probably the cause of twinning planes. Indeed, assuming that the probability of fixation of the twins on the plane $(111)$ is $\mathrm{W}=\exp (-\mathrm{n} \varepsilon / \mathrm{kT})$, where $\mathrm{n}$ is the number of atoms in a flat nucleus and $\varepsilon$ - energy of packing defect per atom, and knowing the ratio of the number of twin planes to normal, equal $10^{-5.5}$, we obtain $\mathrm{W}=\exp (-\mathrm{n} \varepsilon / \mathrm{kt})=$ $10^{-5.5} ; \mathrm{K}=1.4 \times 10^{-16} \mathrm{erg} /$ degree; $\mathrm{T}=1400^{\circ} \mathrm{K}$, and $\mathrm{n} \varepsilon=1.6 \mathrm{eV}$ or $\mathrm{n}=10, \varepsilon=016$ Ev/atom that is reasonable under the order of the values.

The increase in accuracy of temperature regulation promotes some reduction in the number of twins, which also confirms notable influence of fluctuations on the twinning.

Thus, deterioration or shape defects of GaP plates during their growth from Ga-P melt solution are observed in the following cases: 1) considerable increase of concentration of impurities in the GaP solution and dependently on the chemical nature of the chosen impurity; 2) at the change of the crystallization conditions, namely, sufficient decrease of the cooling velocity or in the case of nonuniform heat removal from the container for the crystal growth.

Processes of creation of the dendritic needles and their transformation to platelet crystals take place in a small temperature interval and for a short time, therefore they are hardly controlled, while crystal growth is easy controlled in the direction normal to the plate (111), because it is very sensitive to the external factors such as velocity of the solution cooling and accuracy of the temperature control.

All of the above-stated means that only very perfect growth equipment and top quality experience will give an opportunity to grow good and having high service ability $\mathrm{GaP}$ crystals. However, in spite of the all precautions and high experience of the specialists, growing the crystals, freshly prepared GaP crystals cannot be successfully applied in electronics due to their low operational data, such as absence of high photosensitivity, bright and broadband luminescence, satisfactory transparency and mechanical characteristics. The following sections will demonstrate how to considerably increase the quality and service ability of the freshly prepared GaP crystals.

\section{OPTICAL PROPERTIES OF PERFECT, LONG-TERM ORDERED GAP:N IN COMPARISON WITH FRESH- LY PREPARED CRYSTALS}

Investigating gallium phosphide $(\mathrm{GaP})$ crystals [1, 7] grown by the author in the 1960 s, clear improvement of their optical and mechanical properties were noted for the first time only after 10 to 12 years since the time of their initial preparation. It was unusual and interesting in the situation when all nonliving matter around us usually deteriorates in time. Therefore, the decision was taken to investigate, to understand and to use this phenomenon in the future. In short, the investigation process can be described as follows.

Grown about 50 years ago pure and doped GaP crystals throughout the intervening decades have been periodically re-evaluated in order to see and investigate the change over 


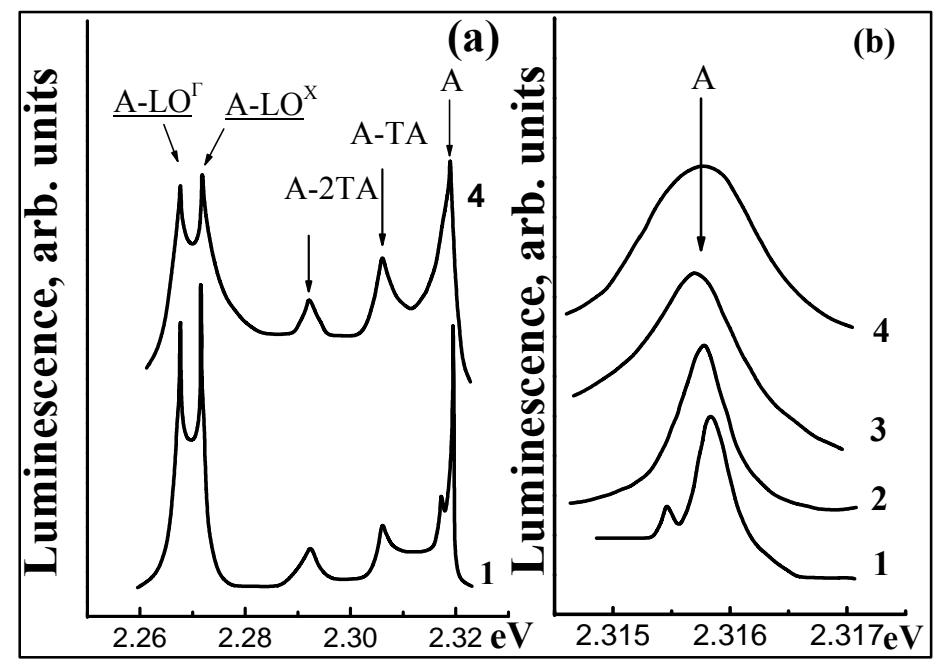

Fig. (4). Evolution of the GaP:N luminescence with time and nitrogen concentration at the temperature of 15K. (a) Zero-phonon line of the bound exciton A and its transversal acoustic (TA) and longitudinal optic (LO) phonon replica in as-prepared (4) and 25-year long-term ordered (1) crystals. (b) Zero-phonon line A as a function of nitrogen $(\mathrm{N})$ concentration. 1-3: 25-year-old crystals. 4: as-prepared. Curves 1 through 4 represent samples with nitrogen concentrations of $10^{17}, 10^{18}, 10^{19}$, and $10^{18} \mathrm{~cm}^{-3}$, respectively.

time in their electro- and photoluminescence, photoconductivity, behavior of bound excitons, giving interesting and bright luminescence, nonlinear optics, and other phenomena. Accordingly, it is of interest also to monitor the change in crystal quality over the course of several decades while the crystal is held under ambient conditions.

Fig. (4) provides a comparison of the evolution in luminescence spectra at low temperatures ( $80 \mathrm{~K}$ and below) from GaP:N over a period of 25 years (original measurement in about 1963). Over this period, zero-phonon line A of single $\mathrm{N}$ impurity-bound excitons and their phonon replica are narrower in their line-widths when compared to the freshly prepared single crystals. Further, as expected, zero phonon line and replica in samples aged at room temperature for 25 years shift spectral position depending upon concentration of $\mathrm{N}$ impurities (Fig. 4b, spectra 1-3) according to [39], while the same freshly prepared crystals exhibited broader luminescence line-widths with increasing nitrogen content (Fig. 4b, spectrum 4). These, along with other half-centennial findings, including modifications in luminescence kinetics, spontaneous Raman scattering, $\mathrm{x}$-ray diffraction, absorption spectra, micro-hardness and density of dislocations, that are reported elsewhere [1, 3-24], strongly suggest that close-toideal GaP:N crystals are forming over time due to the equally-spaced disposition of $\mathrm{N}$ impurities instead of their chaotic distribution in the same freshly prepared crystals.

As first noted in [23], these results suggest a new type of crystal lattice in which the host atoms occupy their proper (equilibrium) positions in the crystal, while the $\mathrm{N}$ impurities, periodically substituted into the lattice portion it into short chains of equal length. According to the data obtained from Raman light scattering $[10,14]$, host atoms of this new lattice develop harmonic vibrations, and high degree of lattice perfection leads to an abrupt decrease in the non-radiative recombination and an increase of efficiency and spectral range of luminescence.

Additionally, stimulated emission of light in these temporally-ordered crystals (Fig. 5b) is observed. As also shown in Ref. [23], the GaP:N crystals aged for at least 40 years possess no discrete impurity level for $\mathrm{N}$-bound excitons in the forbidden gap. They also demonstrated a uniform luminescence from a broad excitonic band instead of the narrow zero-phonon line and its phonon replica as observed from the less-aged, 25 year-old crystals.

Thus, long-term ordered GaP:N crystals demonstrate uniform bright luminescence from a broad excitonic band instead of the narrow zero-phonon line and its phonon replica in disordered and partly ordered (25-year-old) crystals. This is due to the fact that ordered crystals have no discrete impurity level in the forbidden gap. To the best of our knowledge, such transformation of a discrete level within the forbidden gap into an excitonic band (Fig. 5a,b) is observed for the first time. In this case, the impurity atoms regularly occupy the host lattice sites and affect the band structure of the crystals, which is now a dilute solid solution of GaP-GaN with regular disposition of $\mathrm{N}$ atoms instead of freshly prepared $\mathrm{GaP}$ doped by occasionally located $\mathrm{N}$ atoms. Note that the increase of luminescence excitation in case of partly ordered GaP:N (Fig. 5a, dotted line) leads to a broad luminescence band as a result of bound exciton interaction [9], while in the case of perfectly ordered crystals (Fig. 5b) one can see an abrupt narrowing of the luminescence band due to stimulated emission in defect-free crystals. Earlier, in freshly prepared crystals, we observed a clear stimulated emission from a GaP:N resonator at $80 \mathrm{~K} \mathrm{[5],} \mathrm{as} \mathrm{well} \mathrm{as} \mathrm{the} \mathrm{so} \mathrm{called} \mathrm{super-}$ luminescence from $\mathrm{GaP}$ single crystals having natural faceting. Presently, our ordered crystals have a bright luminescence at room temperature that implies their perfection and 


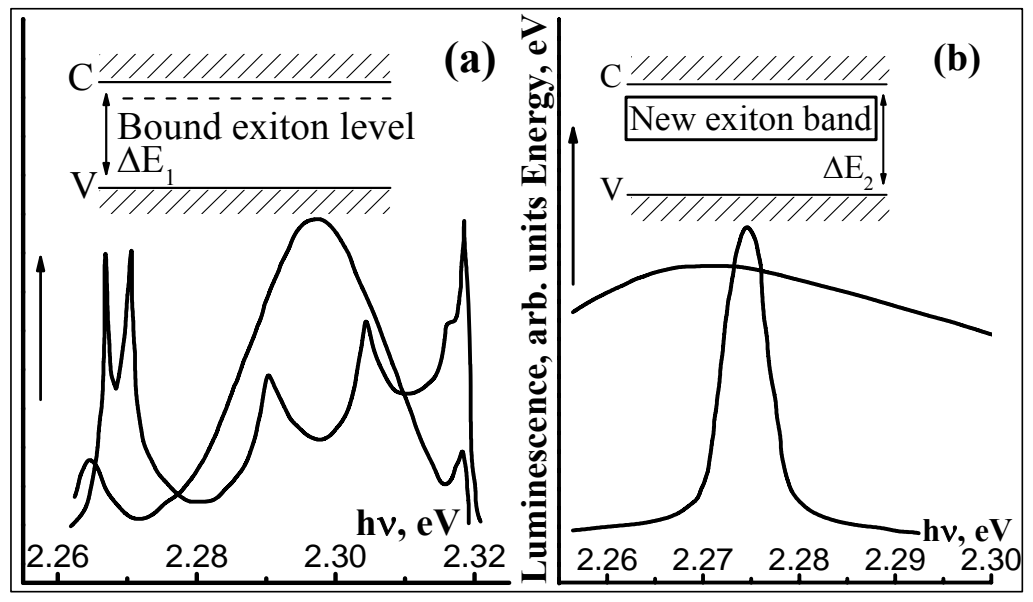

Fig. (5). Luminescent spectra and schematic representation of the forbidden gaps $\left(\Delta \mathrm{E}_{1}, \Delta \mathrm{E}_{2}\right)$ in the nitrogen-doped GaP aged for (a) 25 years and (b) 40 years. The dotted spectra correspond to highly optically excited crystals. $\mathrm{C}$ and $\mathrm{V}$ represent the positions of the bottom of the conductance and the top of valence bands, respectively.

very low light losses. In our studies [14, 23] we demonstrate that the stimulated emission in long-term ordered $\mathrm{GaP}$ is also developed even at room temperature by electron-hole recombination of an electron at the bottom of the conduction band with a hole at the top of the valence band and the LO phonon absorption.

\section{COMPARISON OF OPTICAL PROPERTIES OF GAP NANOCRYSTALS AND GAP PERFECT BULK SIN- GLE CRYSTALS}

It is necessary to note that the very important for optoelectronics long-term ordering and considerable improvement of the semiconductor crystal lattice and accompanying phenomena have been discovered and observed over decade time scales only with the help of grown by the Author unique collection of samples. And up to now this collection of long-term ordered perfect GaP single crystals gives opportunities to find deep fundamental analogies in properties of the perfect single crystals and nanoparticles as well as to predict and to realize in nanoparticles and perfect bulk crystals new interesting properties and applications. Jointly with the references $[3,7,9-17,21-27,31]$ this section is a generalization of the results on long-term observation of luminescence, absorption and Raman light scattering in bulk semiconductors in comparison with some properties of the best to the moment $\mathrm{GaP}$ nanocrystals. Combination of these characterization techniques elucidates the evolution of these crystals over the course of many years, as well as the ordered state brought about by prolonged room-temperature thermal annealing, and the interesting optical properties that accompany such ordering. We demonstrate that long-term natural stimuli result in perfection of our crystals, which can lead to novel heterogeneous systems and new semiconductor devices with high temporal stability.

We further improved upon the preparation of GaP nanocrystals using the known methods of hydrothermal and colloidal synthesis [29-31] taking into account that success of our activity depends on optimal choice of the types of chemical reactions, necessary chemicals and their purity, conditions of the synthesis (control accuracy of preparation, temperature, pressure, duration, etc.), methods and quality of purification of the nanocrystals, storage conditions for nanoparticles used in the further operations of fabrication of the $\mathrm{GaP} /$ nanocomposites. Note, the considerable improvement of quality and luminescent properties of $\mathrm{GaP}$ nanocrystals is the result of elaboration of an optimal for them nanotechnology.

The best quality GaP nanoparticles have been prepared by hydrothermal or colloidal synthesis from white phosphorus at decreased temperature $\left(125^{\circ} \mathrm{C}\right)$ and intense ultrasonication; it was established that the maximum shift to ultraviolet and the best quality in general have the nanocomposites obtained from the nanoparticles stored as a suspension in a suitable liquid.

As we noted earlier, different defects of high concentration in freshly prepared $\mathrm{GaP}$ single crystals completely suppress any luminescence at room temperature due to negligible quantity of free path for non-equilibrium electron-hole pairs between the defects and their non-radiative recombination, while the quantum theory predicts their free movement in the field of an ideal crystal lattice. It was also shown the long-term ordered and therefore close-to-ideal crystals demonstrate bright luminescence and stimulated emission repeating behavior of the best nanoparticles with pronounced quantum confinement effects.

Fig. (6) compares the luminescence spectra of our longterm (up to 50 years) ordered $\mathrm{GaP}$ single crystals (spectrum 1) to that from high quality $\mathrm{GaP}$ nanoparticles and their $\mathrm{GaP}$ nanoparticles/polymers nanocomposites [15, 17]. Nanocrystals stored as dry powder demonstrate rather broad luminescent band with maximum at $2.8 \mathrm{eV}$ (Fig. 6, spectrum 2), while the nanocrystals of about $10 \mathrm{~nm}$ sizes, thoroughly 


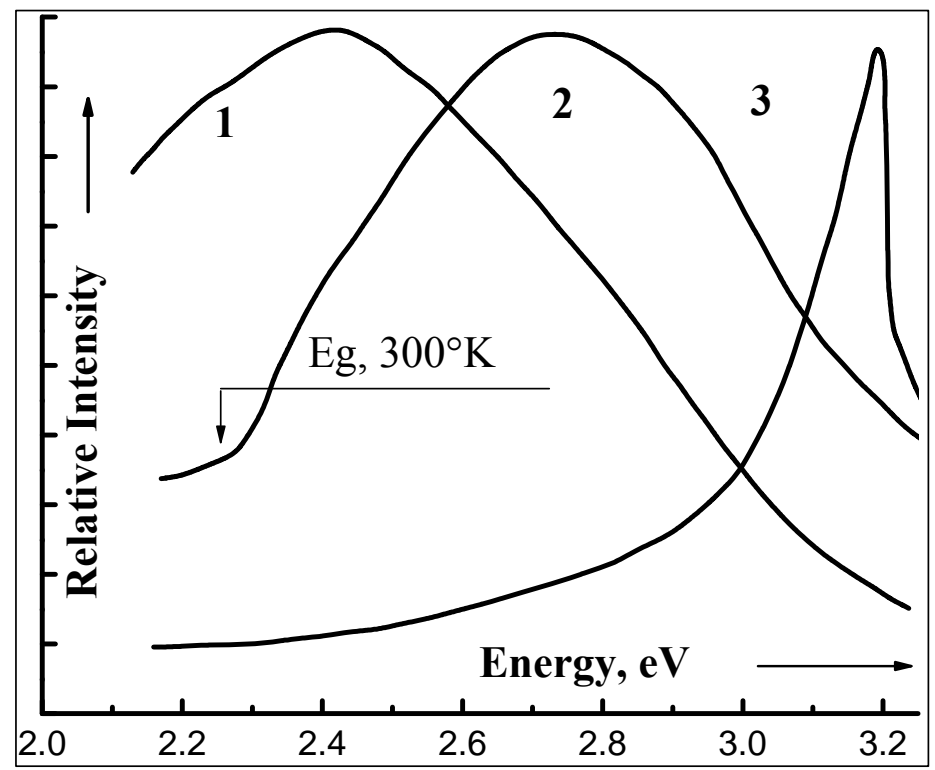

Fig. (6). Luminescence of perfect bulk GaP single crystals (1) in comparison with the luminescence of GaP nanoparticles and GaP/polymers nano-composites $(2,3)$. Nanoparticles were prepared from white $\mathrm{P}$ by mild aqueous or colloidal synthesis at decreased temperature, stored as dry powder (spectrum 2) or suspension in a liquid (spectrum 3). Details: [15, 17].

separated and distributed in a suspension, which prevents their coagulation, mechanical and optical interaction, exhibit bright narrow-band luminescence with maximum at $3.2 \mathrm{eV}$, approximately $1 \mathrm{eV}$ above the position of the absorption edge in GaP at 300oK (Fig. 6, spectrum 3).

The thoroughly washed, ultrasonicated and dried nanopowders as well as their specially prepared suspensions have been used for fabrication of blue light emissive GaP nanocomposites on the base of some optically and mechanically compatible with GaP polymers [15, 17, 29-31]. According to our measurements, the matrix polymers PGMAco-POEGMA or BPVE used in this work provide no contribution to the spectra of luminescence of the based on them matrixes. So, the nanocomposite spectra coincide with those obtained from the relevant $\mathrm{GaP}$ powders or suspensions. We note that in the GaP/BPVE nanocomposite the position of the luminescent maximum can be changed between $2.5-3.2$ $\mathrm{eV}$ and the brightness is $20-30$ more than in the PGMA and PGMA-co-POEGMA matrixes.

We explain the broadening of the luminescence band and the shift of its maximum to low photon energies in luminescence of the nanocomposite based on the GaP powder by presence in the powder of the nanoparticles with the different dimensions between 10-100 nm. Meanwhile, the nanocomposites on the base of the suspensions containing only approximately $10 \mathrm{~nm}$ nanoparticles, exhibit bright luminescence with maximum at $3.2 \mathrm{eV}$ due to high transparency of $10 \mathrm{~nm}$ nanoparticles for these high energy emitted photons and pronounced quantum confinement effect.

In order to explain this interesting phenomenon, we postulate that the nanocrystals, much like the ideal long-term ordered bulk GaP single crystals, exhibit this huge increase in blue-shifted luminescence due to: (a) negligibly small influence of defects and non-radiative recombination of electron-hole pairs and very high efficiency of their radiative annihilation, (b) high perfection of nanocrystal lattice, and (d) high transparency of nanocrystals due to their small dimensions for the light emitted from high points of the $\mathrm{GaP}$ Brillouin zones, for instance, in the direct transitions $\Gamma_{1}{ }^{\mathrm{c}}$ $\Gamma_{15}{ }^{\mathrm{v}}$ between the conductive and valence bands with the photon energy at $300 \mathrm{~K}$ equal to $2.8 \mathrm{eV}$ [36] and (e) high efficiency of this so called "hot" luminescence.

Our first attempts to prepare $\mathrm{GaP}$ nanoparticles [18, 27] yielded room temperature luminescence with maximum shifted only to $2.4 \mathrm{eV}$ in comparison with the achieved now new maximum at $3.2 \mathrm{eV}$ achieved today. It confirms significant progress in technology of $\mathrm{GaP}$ nanoparticles and $\mathrm{GaP} /$ polymers nanocomposites. On the base of these improved technologies for preparation of $\mathrm{GaP}$ nanoparticles and $\mathrm{GaP} /$ polymer nanocomposites we can change main parameters of luminescence within the broad limits, and to create a framework for novel light emissive device structures using dramatic $1 \mathrm{eV}$ expansion of $\mathrm{GaP}$ luminescence to $\mathrm{UV}$ region.

\section{EXCITONIC CRYSTAL AND ITS IMPORTANCE IN OPTOELECTRONICS}

The role and application of bound excitons in nanoscience and technology is discussed in this chapter. Bound excitons are well studied in semiconductors, especially in gallium phosphide doped by nitrogen $(\mathrm{GaP}: \mathrm{N})$. Doping of $\mathrm{GaP}$ with $\mathrm{N}$ leads to isoelectronic substitution of the host $\mathrm{P}$ atoms by $\mathrm{N}$ in its crystal lattice and to the creation of the electron trap with a giant capture cross-section. Therefore, any nonequilibrium electron in the vicinity of the trap will be captured by $\mathrm{N}$ atom, attracting a non-equilibrium hole by 


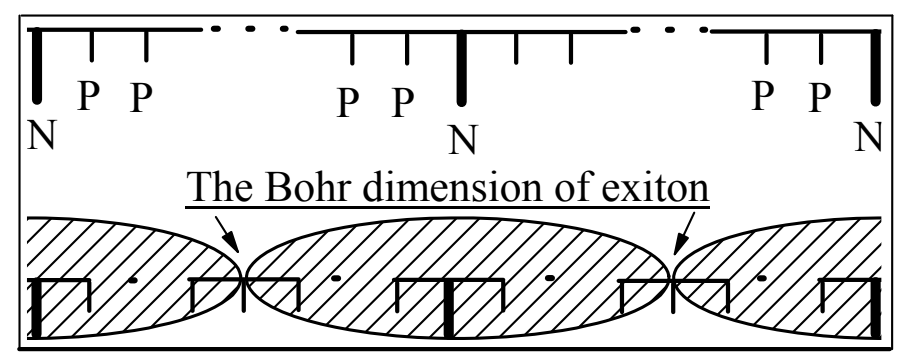

Fig. (7). The models of the well-ordered GaP:N. a. The new type of crystal lattice with periodic substitution of $\mathrm{N}$ atoms for the host $\mathrm{P}$ atoms. b. The excitonic crystal on the base of this lattice. The substitution period is equal to the Bohr diameter of exciton $(\sim 100 \AA)$ and optical excitation is enough for complete saturation of the $\mathrm{N}$ sublattice with non-equilibrium electron-hole pairs (please, see details in [15, 17].

Coulomb interaction and creating the bound exciton - shortlived nanoparticle with the dimension of the order of $10 \mathrm{~nm}$ (it is the Bohr diameter of bound exciton in GaP:N). Note, that none of the nanotechnology methods are used in creation or selection of dimensions of these nanoparticles - only natural forces of electron-hole interaction and electron capture by the traps are necessary for the creation of these nanoparticles. As the result we get something like neutral shortlived atom analogue - a particle consisting of heavy negatively charged nucleus ( $\mathrm{N}$ atom with captured electron) and hole. So called "zero vibrations" do not destroy possible solid phase of bound excitons having these heavy nuclei that gives an opportunity to reach their crystal state - short-lived excitonic crystal.

Taking into account the above-mentioned results, a model for the crystal lattice and its behavior at a high level of optical excitation for well-ordered N-doped GaP (Fig. 7) can be suggested. At the relevant concentrations of $\mathrm{N}$, the anion sub-lattice can be represented as a row of anions where $\mathrm{N}$ substitutes $\mathrm{P}$ atoms with the period equal to the Bohr diameter of the bound exciton in GaP (approximately $10 \mathrm{~nm}$ ) (Fig. 7a). At some level of excitation, all the $\mathrm{N}$ sites will be filled by excitons, thereby creating an excitonic crystal (Fig. 7b) which is a new phenomenon in solid-state physics and a very interesting medium for application in optoelectronics and nonlinear optics $[4,15,17,25,26]$.

Thus, using bound excitons as short-lived analogues of atoms and sticking to some specific rules, including the necessity to build the excitonic superlattice with the identity period equal to the bound exciton Bohr dimension in the $\mathrm{GaP}: \mathrm{N}$ single crystal, we get a unique opportunity to create a new solid state media - consisting from short-lived nanoparticles excitonic crystal, obviously, with very useful and interesting properties for application in optoelectronics, nanoscience and technology. The following will discuss methods of preparation and possible application of $\mathrm{GaP}$ excitonic crystals and nanocrystals in optoelectronics.

\section{CONCLUSION}

Since the time of original preparation of gallium phosphide doped by nitrogen crystals (GaP:N) by the author in the 1960 s, followed by the introduction of the excitonic crystal concept in the 1970s, the best methods of bulk, film and nanoparticle crystal growth were elaborated. The results of semi centennial evolution of $\mathrm{GaP}: \mathrm{N}$ properties are compiled here and in the references to this review. Novel and useful properties of $\mathrm{GaP}$ including an expected similarity in behavior between nanoparticles and perfect bulk crystals, as well as very bright and broadband luminescence at room temperature, are observed.

These results provide a new approach to selection and preparation of perfect materials for optoelectronics [25] and a unique opportunity to realize a new form of solid-state host - the excitonic crystal [26]. In spite of the fact that the time necessary for natural long-term ordering (years) does not lead to optimism, the experience and results collected here confirm expedience of the efforts directed to formation of the $\mathrm{N}$ impurity superlattice in $\mathrm{GaP}$ having the identity period equal to the bound exciton dimension. As noted in [25], the process of preparation of top quality material for industrial electronics can be organized if the freshly grown crystals will be kept for years in a special storage and only old crystals (like to the old wine, if to use close to Moldova and other winemaking countries analogy) with the necessary properties will be annually retrieved for device fabrication while new portions of fresh crystals will be placed for long-term ordering.

Except natural aging of the relevant crystals for years, preparation of the $\mathrm{N}$ superlattice for excitonic crystal can be also realized by known methods of growth of multi-layer films, in particular, by molecular beam and laser assisted epitaxy [40].

The excitonic crystal, created by the long-term ordering or by the noted above methods of growth of multi-layer films, as well as the bulk top quality GaP crystals with the unique optic properties, obtained by the long-term ordering process of freshly prepared crystals, will be used in the new generation of optoelectronic devices, sometimes instead of nanoparticles and a lot of other materials. In particular, keeping in mind the low energy of the bound exciton creation, one can expect a low threshold for generation of non-linear optical effects in the excitonic crystal and a good opportunity to create new and very efficient optoelectronic devices.

Independently on their dimensions, the perfect GaP bulk single crystals and its nanoparticles demonstrate very interesting for application properties. Therefore, using the 
long-term ordered, perfect $\mathrm{GaP}$ crystals or similar on behavior and properties material in electronic industry instead of elaboration of very expensive and labor-consuming technologies for diverse materials and their nanoparticles with limited for application spectral region and other parameters, we get a big commercial advantage from their fabrication and application [for more details please see the paper [25].

The unique collection of pure and doped crystals of semiconductors grown in the 1960s provides an opportunity to observe the long-term evolution of properties of these key electronic materials. During this half-centennial systematic investigation we have established the main trends of the evolution of their optoelectronic and mechanical properties. It was shown that these stimuli to improve quality of the crystal lattice are the consequence of thermodynamic driving forces and prevail over tendencies that would favor disorder.

For the first time, to the best of our knowledge, we have observed a new type of the crystal lattice where the host atoms occupy their proper (equilibrium) positions in the crystal field, while the impurities, once periodically inserted into the lattice, divide it in the short chains of equal length, where the host atoms develop harmonic vibrations. This periodic substitution of a host atom by an impurity allows the impurity to participate in the formation of the crystal's energy bands. In $\mathrm{GaP}$ it leads to the change in the value of the forbidden energy gap, to the appearance of a crystalline excitonic phase, and to the broad excitonic energy bands instead of the energy levels of bound excitons. Perfection of this new lattice sharply decreases non-radiative electron-hole recombination, increases the efficiency and spectral range of luminescence and promotes the stimulated emission of light due to its amplification inside the well-arranged, defect-free medium of the crystal. Further development of techniques for growth of thin films and bulk crystals with ordered distribution of impurities and proper localization of host atoms inside the lattice should be of high priority.

Our long-term ordered and therefore close to ideal crystals repeat behavior of the best nanoparticles with pronounced quantum confinement effect [15].

Especially important for application in new generation of light emissive devices are:

1) Predicted and investigated by us crystalline state of bound excitons in GaP:N;

2) Dramatic expansion of luminescence region in $\mathrm{GaP}$ perfect bulk single crystals [16, 31], and

3) The best prepared GaP nanocrystals and based on them composites with transparent polymers.

So, the results of this long-term evolution of the important properties of our unique collection of semiconductor single crystals promise a novel approach to the development of a new generation of optoelectronic devices.

Besides the long-term ordering, the combined methods of laser assisted and molecular beam epitaxies [40-42] will be applied to fabrication of device structures with artificial periodicity; together with classic methods of perfect crystal growth, they can be employed to realize impurity ordering that would yield new types of nanostructures and enhanced optoelectronic device performance.

For the first time we demonstrate that well-aged GaP bulk crystals as well as high quality GaP nanoparticles have no essential difference in their luminescence behavior, brightness or spectral position of the emitted light. These perfect crystals are useful for application in top-quality optoelectronic devices as well as they are a new object for development of fundamentals of solid state physics.

Of course, waiting for improvement of crystal quality for decades can be justified only in exceptional cases, but we propose to turn this perennial procedure of long-term ordering into the necessary technique for preparation of the top quality material for industrial electronics.

\section{CONFLICT OF INTEREST}

The author confirms that the content of this review has no conflicts of interest.

\section{ACKNOWLEDGEMENTS}

I am glad to note, that the broad discussion and dissemination of our joint results stimulates further collaboration with reliable partners from the USA, Russia, Italy, Romania, France and other countries. I express my cordial gratitude to my teachers, world-known scientists late Profs Nina A. Goryunova, Nobel Prize Laureate Alexander M. Prokhorov, Rem V. Khokhlov and Sergei I. Radautsan. The author is very grateful to the US Department of State, Institute of International Exchange, Washington, DC, The US Air Force Office for Scientific Research, the US Office of Naval Research Global, Civilian R\&D Foundation, Arlington, VA, Science \& Technology Center in Ukraine, to his colleagues and co-authors from Clemson University, SC, University of Central Florida, FL, Istituto di elettronica dello stato solido, CNR, Rome, Italy, Universita degli studi, Cagliari, Italy, Lomonosov Moscow State University, Ioffe PhysicoTechnical Institute and State Polytechnical University Ioffe, St. Petersburg, Ac. Scie. Institute of General Physics, Moscow, Russia, Institute of Applied Physics and Academy of Sciences of Moldova for support and attention to this protracted (1963-present time) research.

\section{REFERENCES}

[1] Goryunova NA, Pyshkin SL, Borshchevskii AS, et al. Influence of impurities and crystallization conditions on growth of platelet $\mathrm{GaP}$ crystals. J Growth of Crystals 1969; 8: 68-72, ed. N.N. Sheftal, New York.: Symposium on Crystal Growth at the $7^{\text {th }}$ International Crystallography Congress (Moscow, July 1966).

[2] Pyshkin SL, Radautsan SI, Zenchenko VP. Processes of longlasting ordering in crystals with a partly inverse spinel structure. Sov Phys - Dokl 1990; 35(4): 301-4.

[3] Pyshkin SL. Preparation and properties of gallium phosphide. Ph.D. thesis. Kishinev: State University of Moldova 1967.

[4] Pyshkin SL, Zifudin L. Excitons in highly optically excited gallium phosphide. J Lumin 1974; 9: 302-8. 
[5] Pyshkin SL. Stimulated emission in gallium phosphide. Sov PhysDokl 1975; 19: 845-6.

[6] Pyshkin S. Luminescence of GaP: N: Sm Crystals. J Soviet Phys Semicond 1975; 8: 912-3.

[7] Pyshkin SL. Photoconductivity and luminescence of highly optically excited semiconductors. Dr. Sc. thesis. Russia: Lomonosov Moscow State University 1978.

[8] Pyshkin S, Anedda A, Congiu F, Mura A. Luminescence of the GaP:N Ordered System. J Pure Appl Opt 1993; 2: 499-502.

[9] Pyshkin SL, Anedda A. Time-dependent behaviour of antistructural defects and impurities in Cd-In-S and GaP. Institute of Physics Conference Series, Ternar Multin Comp 1998; 152, Section E: 7859.

[10] Pyshkin S, Ballato J, Chumanov G. Raman light scattering from long-term ordered GaP single crystals. J Opt Pure Appl Opt 2007; 9: 33-6.

[11] Pyshkin SL, Ballato J, Bass M, Turri G. Luminescence of longterm ordered pure and doped gallium phosphide. TMS Annual Meeting, invited talk at the Symposium: advances in Semiconductor, Electro Optic and Radio Frequency Materials, March 9-13, New Orleans, LA. J Electron Mater 2008; 37(4): 388-95.

[12] Pyshkin SL, Ballato J, Bass M, Chumanov G. Time-dependent evolution of crystal lattice, defects and impurities in $\mathrm{CdIn}_{2} \mathrm{~S}_{4}$ and GaP. J Phys Status Sol 2009; C(6): 1112-5.

[13] Pyshkin SL, Ballato J, Bass M, Turri G. Evolution of luminescence from doped gallium phosphide over 40 Years. J Electron Mater 2009; 38(5): 640-6.

[14] Pyshkin SL, Ballato J. Evolution of optical and mechanical properties of semiconductors over 40 Years. J Electron Mater 2010; 39(6): 635-41.

[15] Pyshkin SL, Ballato J. Long-term convergence of bulk- and nanocrystal properties. In: Optoelectronics - Materials and Technics, InTech - 2011; Chapter 19; pp. 459-76.

[16] Pyshkin SL, Ballato J. Dramatic expansion of luminescence region in $\mathrm{GaP} /$ polymer nanocomposites. Supplemental Proceedings of the 2012 TMS Annual Meetings. Orlando, FL, USA, March 11-15, Materials Processing and Interfaces 2012; vol 1: pp. 353-9.

[17] Pyshkin SL, Ballato J. Advanced light emissive device structures. In: Optoelectronics - Advanced Materials and Devices. Pyshkin SL, and Ballato J. eds. InTech; Coartia 2013.

[18] Pyshkin SL, Ballato J, Chumanov G, DiMaio J, Saha AK. Preparation and characterization of nanocrystalline GaP. Technical Proceedings of the 2006 NSTI Nanotech Conference; vol. 3: pp. 194-7.

[19] Pyshkin S, Ballato J, Chumanov G, Tsyntsaru N, Rusu E. Preparation and characterization of nanocrystalline $\mathrm{GaP}$ for advanced light emissive device structures. In: Proceedings of the NSTI-Nanotech Conference (Anaheim, CA) 2010; vol. 1: pp. 522-5.

[20] Pyshkin SL, Ballato J, Belevschii S, Rusu E, Racu A, Van DerVeer D. Synthesis and characterization of GaP nanoparticles for light emissive devices. In: Proceedings of the NSTI-Nanotech Conference, June 13-16. Boston 2011; vol. 1: pp. 327-30.

[21] Pyshkin SL. Excitonic crystal, nanotechnology and new prospect for optoelectronics. $7^{\text {th }}$ International Conference on Materials Science and Condensed Matter Physics. September 16-19, Kishinev: Moldova Abstract Book, p. 214, 2014.

[22] Pyshkin S, Zhitaru R, Ballato J, Chumanov G, Bass M. Structural characterization of long-term ordered semiconductors. Proceedings of the MS\&T Conference, International Symposium, Fundamentals \& Characterization 2009; pp. 698-709.
[23] Pyshkin S, Ballato J, Bass M, Chumanov G, Turri G. Properties of the long-term ordered semiconductors. Proceedings of the 2009 TMS Annual Meeting and Exhibition; San Francisco 2009.

[24] Pyshkin S, Ballato J, Chumanov G, et al. Optical and mechanical properties of long-term ordered semiconductors. MJPS 2009; 8(34): $287-95$.

[25] Pyshkin SL. Gallium Phosphide - new prospect for optoelectronics. J Adv Optoelectron Mater 2013; 1(4): 59-66.

[26] Pyshkin SL. Excitonic crystal and nanotechnology. Nanosci Technol 2014; 1(2): 1-5. (Available from http://symbiosisonlinepublishing. com/nanosciencetechnology08.pdf)

[27] Pyshkin SL, Ballato J. Advanced light emissive composite materials for integrated optics. Symposium: The Physics and Materials Challenges for Integrated Optics - A Step in the Future for Photonic Devices. Proceedings of the MS\&T Conference, Pittsburgh 2005; pp. 3-13.

[28] Pyshkin SL, Ballato J, Luzinov I, Zdyrko B. Fabrication and characterization of the GaP/polymer nanocomposites for advanced light emissive device structures. The NSTI-Nanotech Conference 2010; vol. 1: pp. 772-5.

[29] Pyshkin S, Ballato J, Chumanov G, Tsyntsaru N, Rusu E. Preparation and characterization of nanocrystalline $\mathrm{GaP}$ for advanced light emissive device structures. The NSTI-Nanotech Conference 2010; vol. 1: pp. 522-5.

[30] Pyshkin SL, Ballato J, Luzinov I, Zdyrko B. Fabrication and characterization of $\mathrm{GaP} /$ polymer nanocomposites for advanced light emissive device structures. J Nanopart Res 2011; 13: 5565-70.

[31] Pyshkin SL. Advanced Light Emissive Device Structures. STCU (www.stcu.int) Project 4610, 2009-2012.

[32] Gao S, Lu J, Chen N, Zhao Y, Xie Y. Aqueous synthesis of iii-v semiconductor $\mathrm{GaP}$ and $\mathrm{InP}$ exhibiting pronounced quantum confinement. Chem Commun 2002; 21(24): 3064-5.

[33] Liu Z, Bai Y, Cui D, Wang Q. Preliminary insight into the formation process of InP and GaP nanocrystals. Solid State Sci 2003; 5: $1037-40$.

[34] Zhang Z-C, Wang B-P. Diamond and oxidized disordered graphite on the surface of gallium phosphide nanoparticles. Syst Charact 2009; 26: 53-7.

[35] Paul W. Band structure of the intermetallic semiconductors from pressure experiments. J Appl Phys 1961; 32: 2082-94.

[36] Zallen R, Paul W. Band structure of gallium phosphide from optical experiments at high pressure. Phys Rev 1964; 134: A1628-41.

[37] Dean PJ, Thomas DG. Intrinsic absorption-edge spectrum of gallium phosphide. Phys Rev 1966; 150(2): 690-702.

[38] Kleinman DA, Spitzer WG. Infrared lattice absorption of GaP. Phys Rev 1960; 118 (1): 110-7.

[39] Allen WJ. Energy levels of nitrogen-nitrogen pairs in Gallium Phosphide. J Phys, C: Solid State Phys 1968; 1: 1136-8.

[40] Pyshkin SL, Fedoseev SA, Lagomarsino S, Giannini C. preparation and structural properties of some III-V semiconductor films grown on (100) oriented si substrates. J Appl Surf Sci 1992; 56-58: 39-43.

[41] Budyanu VA, Chechuy SN, Pyshkin SL, et al. Investigation of IIIV/Si heterojunction grown by laser deposition. Phys Stat Sol 1985; 91: 737-44.

[42] Budyanu VA, Chechuy SN, Pyshkin SL, et al. Laser vacuum epitaxy of III-V semiconductors on silicon. Rev Roumaine de Physique 1987; 32(1-2): 216-19.

This is an open access article licensed under the terms of the Creative Commons Attribution Non-Commercial License (http://creativecommons.org/licenses/by$\mathrm{nc} / 3.0 /$ ) which permits unrestricted, non-commercial use, distribution and reproduction in any medium, provided the work is properly cited. 